\title{
A NOTE ON THE GEOMETRIC ERGODICITY OF A MARKOV CHAIN
}

\author{
K. S. CHAN, ${ }^{*}$ University of Chicago
}

\begin{abstract}
It is known that if an irreducible and aperiodic Markov chain satisfies a 'drift' condition in terms of a non-negative measurable function $g(x)$, it is geometrically ergodic. See, e.g. Nummelin (1984), p. 90. We extend the analysis to show that the distance between the $n$ th-step transition probability and the invariant probability measure is bounded above by $\rho^{n}(a+$ $b g(x))$ for some constants $a, b>0$ and $\rho<1$. The result is then applied to obtain convergence rates to the invariant probability measures for an autoregressive process and a random walk on a half line.
\end{abstract}

AUTOREGRESSIVE PROCESS; DRIFT CONDITION; RANDOM WALK

\section{Introduction}

Let $(R, \mathscr{E})$ be a measurable space, $\mathscr{E}$ being assumed countably generated. Let $\phi$ be a non-trivial $\sigma$-finite measure on $(E, \mathscr{E})$. An $E$-valued Markov chain $\left(X_{n}\right)_{n \in \mathbb{N}}$ is said to be $\phi$-irreducible if

$$
\Sigma_{0 \leqq n<\infty} P^{n}(x, A)>0 \text { for all } A \in \mathscr{E} \text { with } \phi(A)>0,
$$

where $P(.,$.$) denotes the transition probability. In Sections 1$ and $2,\left(X_{n}\right)$ is assumed to be a $\phi$-irreducible Markov chain. $\left(X_{n}\right)$ is said to be geometrically ergodic if it admits an invariant probability measure $\pi$, a $\pi$-integrable function $M$ and a constant $\rho<1$ such that

$$
\left\|P^{n}(x, .)-\pi\right\| \leqq M(x) \rho^{n}, \quad \forall x \in E, \quad n \geqq 0,
$$

where $\|$.$\| denotes the total variation norm.$

It is known that if $\left(X_{n}\right)$ is aperiodic and satisfies a 'drift' condition in terms of a well-behaved non-negative measurable function $g($.$) , then it is geometrically ergodic. We$ extend the analysis to show that $M(x)$ in (1.2) can be taken as $a+b g(x)$ for some positive constants $a$ and $b$. We follow the notation adopted in Nummelin (1984) and refer the reader to it for any unexplained notation.

\section{Main result}

We now state the main theorem.

Theorem 1. Suppose that $\left(X_{n}\right)$ is aperiodic and that for some small set $C$, a non-negative measurable function $g$, a constant $r>1$ such that

$$
\begin{aligned}
& \sup _{x \in C^{c}} \mathbb{E}\left(r g\left(X_{n+1}\right)-g\left(X_{n}\right) \mid X_{n}=x\right) \equiv \gamma<0 ; \\
& \sup _{x \in C} \mathbb{E}\left(g\left(X_{n+1}\right) ; X_{n+1} \in C^{c} \mid X_{n}=x\right) \equiv B<\infty ; \\
& g(x) \text { is bounded away from } 0 \text { and }+\infty \text { on } C .
\end{aligned}
$$

Then $\left(X_{n}\right)$ is geometrically ergodic and $M(x)$ in (1.2) can be taken as $a+b g(x)$ for some constants $a$ and $b$.

Received 23 April 1988; revision received 4 May 1989.

${ }^{*}$ Postal address: Department of Statistics, The University of Chicago, Chicago, IL 60637, USA. 
Before we prove the theorem, first some remarks. Suppose that condition (2.1) hold. Then Proposition 5.21 in Nummelin (1984) shows that $\left(X_{n}\right)$ is geometrically recurrent. Thus if $\left(X_{n}\right)$ is aperiodic, then it is geometrically ergodic. It also follows from Theorem 4 in Tweedie (1983) that $g($.$) is \pi$-integrable. Here, we extend the analysis to show that actually $M(x)$ in (1.2) can be taken as $a+b g(x)$ for some positive constants $a$ and $b$.

Condition (2.1) is referred to as a kind of 'drift' condition in the literature.

Proof of Theorem 1. It follows from the smallness of $C$ that there exist an integer $m_{0}$, a constant $1 \geqq \beta>0$, a probability measure $v$ such that

$$
P^{m_{0}}(x, A) \geqq \beta 1_{C}(x) v(A), \quad \forall x \in E, \quad A \in \mathscr{E} .
$$

Let $\beta 1_{c}(x)$ be denoted by $s(x)$. The pair $(s, v)$ is called an atom and is denoted by $\alpha$. Since $\left\|P^{n}(x,)-.\pi\right\|$ is non-increasing in $n$, with no loss of generality, we can assume that $m_{0}=1$ lest we work with $\left(X_{n m_{0}}\right)$.

Arguing as in the proof of Theorem 6.14 in Nummelin (1984), we have

$$
\begin{aligned}
\Sigma_{n} r^{n}\left\|P^{n}(x, .)-\pi\right\| \leqq & G_{\alpha}^{(r)} 1(x)+r G_{\alpha}^{(r)} s(x) \Sigma_{m} r^{m}\left|u_{m}-M_{b}^{-1}\right| v G_{\alpha}^{(r)} 1 \\
& +r(r-1)^{-1} \pi(s) v G_{\alpha}^{(r)} 1\left(G_{\alpha}^{(r)} s(x)+1\right),
\end{aligned}
$$

all summations being from 0 to $\infty$.

It follows from condition (2.1) and the proof of Proposition 5.21 in Nummelin (1984) that, after some arrangement,

$$
\mathbb{E}_{x}\left(r^{s_{C}}\right) \leqq a_{1}+b_{1} g(x)
$$

where $a_{1}$ and $b_{1}$ can be chosen as $r((r-1) B / \gamma+1)+1+r$ and $(r-1) / \gamma$ respectively.

From (2.2), we see that there is a probability $\beta>0$ that $X_{n} \in \alpha$ given that $X_{n} \in C$. Thus, in view of the smallness of $C$, it follows from Lemma 5.6 in Nummelin that there exists a constant $a_{2}$ such that

$$
\mathbb{E}_{x}\left(r^{s_{\alpha}}\right) \leqq a_{2} \mathbb{E}_{x}\left(r^{s_{C}}\right)=a_{1} a_{2}+b_{1} a_{2} g(x)
$$

Now,

$$
G_{\alpha}^{(r)} s(x)=\mathbb{E}_{x}\left(r^{T_{\alpha}}\right) \leqq \mathbb{E}_{x}\left(r^{s_{\alpha}}\right) .
$$

Applying Lemma 6.2 in Nummelin (1984) with $\lambda$ there chosen as $\varepsilon_{x}$, the probability measure with all its mass at $x$, we have

$$
G_{\alpha}^{(r)} 1(x)<(r-1)^{-1} G_{\alpha}^{(r)} s(x) .
$$

Now arguing again as in the proof of Theorem 6.14 in Nummelin (1984), by decreasing $r>1$ if necessary, we have both $\Sigma_{m} r^{m}\left|u_{m}-M_{b}^{-1}\right|$ and $v G_{\alpha}^{(r)} 1$ being finite. Combining (2.3), (2.5), (2.6) and (2.7), it is readily seen that there exist positive constants $a$ and $b$ such that $\Sigma_{n} r^{n}\left\|P^{n}(x,)-.\pi\right\| \leqq a+b g(x)$. So, by taking $\rho=r^{-1}$, the proof of the theorem is completed.

\section{Examples}

Theorem 1 provides an upper bound on the convergence rate to the invariant probability measure in the form of $\rho^{n}(a+b g(x))$ with $\rho<1$. We now consider two examples in which $g(x)$ may be chosen as linear in $|x|$ and exponential in $x$ respectively. It is also noted that the convergence rate in $x$ thus obtained is exact for some special cases.

Example 1. Let $\left(X_{n}\right)$ be the stable first-order autoregressive process, i.e.,

$$
X_{n}=\phi X_{n-1}+a_{n}, \quad n=1,2,3, \cdots
$$

where $|\phi|<1, \quad\left(a_{n}\right)$ i.i.d. with finite first absolute moment and $a_{n}$ independent of $X_{n-1}, X_{n-2}, \cdots, X_{0}$. It is assumed that $a_{1}$ has an absolutely continuous component which admits a density positive over some open interval about 0 . Then $\left(X_{n}\right)$ is irreducible and 
aperiodic. Let $g(x)=|x|+1$. Then condition (2.1) holds with $C$ chosen as $[-c, c]$ for some $c>0$. It follows from Theorem 1 that an upper bound on the rate of convergence to the invariant probability measure is linear in $|x|$. In the case of $a_{n}$ being Gaussian, it can be directly verified that the rate of convergence is indeed linear in $|x|$.

Example 2. Let $\left(X_{n}\right)$ be a random walk on $\mathbb{R}^{+}$, i.e.,

$$
X_{n+1}=\left(X_{n}+a_{n}\right)^{+}, \quad n=1,2,3, \cdots
$$

where $\left(a_{n}\right)$ is i.i.d.; $a_{n}$ independent of $X_{n-1}, X_{n-2}, \cdots, X_{0} ; E\left(a_{1}\right)<0$ and, for some $M<\infty$ and $\beta>0, \operatorname{Pr}\left(a_{1}>y\right) \leqq M \exp (-\beta y), \forall y>0$. Then $\left(X_{n}\right)$ is aperiodic and irreducible. It is shown in Nummelin and Tuominen (1982) that condition (2.1) holds with $g(x)=\exp (t x)+1$ for some positive $t$ and $C$ chosen as $[0, c]$ for some $c>0$. It follows from Theorem 1 that an upper bound on the convergence rate to the invariant probability measure is exponential in $x$. In the special case when

$$
a_{1}=\left\{\begin{aligned}
-1 & \text { with probability } p \\
0 & \text { with probability } q
\end{aligned}\right.
$$

with $p+q=1$ and $0<p<1$, it is readily seen that the rate of convergence is indeed exponential in $x$.

\section{References}

Nummelin, E. (1984) General Irreducible Markov Chains and Non-negative Operators. Cambridge University Press.

Nummelin, E. AND TuOMinen, P. (1982) Geometric ergodicity of Harris recurrent Markov chains with applications to renewal theory. Stoch. Proc. Appl. 12, 187-202.

TweEdie, R. L. (1983) The existence of moments for stationary Markov chains. J. Appl. Prob. 20, 191-196. 